VOL. 51 (1995) [163-169]

\title{
AREA-DIAMETER AND AREA-WIDTH RELATIONS FOR COVERING PLANE SETS
}

\section{Salvatore Vassallo}

\begin{abstract}
An area-diameter relation and an area-width relation for plane lattice-point-freeconvex bodies is proved. This implies relations on covering sets with respect to general lattices.
\end{abstract}

\section{INTRODUCTION AND NOTATIONS}

Let $E^{2}$ denote the Euclidean plane and let $\mathcal{L}^{2}$ denote the set of lattices $L \subset E^{2}$ with $\operatorname{det}(L) \neq 0$. Further let $\mathcal{K}^{2}$ denote the set of convex bodies $K \subset E^{2}$. For $K \in \mathcal{K}^{2}$, let $A(K), D(K)$ and $\Delta(K)$ be the area, the diameter and the minimal width of $K$ respectively. Further for $L \in \mathcal{L}^{2}$ let $\lambda_{i}(L)$ be the successive minima of $L$, that is, $\lambda_{i}(L)=\lambda_{i}\left(B^{2}, L\right)=\min \left\{\lambda>0 \mid \operatorname{dim} \operatorname{aff}\left(\lambda B^{2} \cap L\right) \geqslant i\right\}$ and let $\mu_{i}(L)$ be the covering minima of the lattice $L$, that is, $\mu_{i}(L)=\mu_{i}\left(B^{2}, L\right)=\min \left\{\mu>0 \mid \mu B^{2}+\mathbf{g}, \mathbf{g} \in L\right.$, meets every flat $F$ of $E^{2}$ with $\left.\operatorname{dim}(F)=2-i\right\}$ (for these definitions see [4] and [5]).

Note that $\lambda_{1}(L)$ is the length of the shortest non-zero vector of $L$ and $2 \mu_{1}(L)$ is the maximal distance of two adjacent lattice lines. Therefore $\operatorname{det}(L)=2 \mu_{1} \lambda_{1}$ and $2 \mu_{1}(L)=1 / \lambda_{1}\left(L^{*}\right)$ where $L^{*}$ is the reciprocal lattice of $L$. The relation $2 \mu_{1} \geqslant \sqrt{3} / 2 \lambda_{1}$ will be also useful in the sequel (see, for example, [4]).

Let $G(K, L)=$ card $((\operatorname{int} K) \cap L)$ denote the lattice point enumerator.

A convex set $K$ is called a lattice-point-free convex set with respect to $L$, if $G(K, L)=0$. Further $K$ is a covering set if $K+L=\{K+\mathbf{g} \mid \mathbf{g} \in L\}=E^{2}$.

For the integer lattice $\mathrm{Z}^{2}$ there are several inequalities relating $\Delta(K), D(K)$, $A(K)$ and the perimeter $P(K)$ of covering sets or lattice-free convex bodies (see [2]); but only a few results concerning arbitrary lattices $[6,7,10,11]$.

In this paper we generalise two results of $\operatorname{Scott}[8,9]$ to arbitrary lattices.

\section{Results}

Let us denote by $\tau$ the unique solution of the equation $\int_{0}^{t} \sqrt{1-x^{2}} d x=\pi / 8(\tau \simeq$ 0.403977 and $\tau=\sin \left(\phi^{*}\right)$, where $\phi^{*}$ is, as in Scott's theorem [8], the unique solution of $\sin (2 \phi)+2 \phi=\pi / 2)$ then we get:

Received 4th May, 1994

Work partially supported by M.U.R.S.T. $(40 \%-60 \%)$.

Copyright Clearance Centre, Inc. Serial-fee code: 0004-9729/95 \$A2.00+0.00. 
Theorem 1. If $K \in \mathcal{K}^{2}$ and $L \in \mathcal{L}^{2}$, with $G(K, L)=0$, then

$$
\frac{A(K)}{D(K)} \leqslant \max \left\{2 \mu_{1}(L), 2 \tau \sqrt{\lambda_{1}^{2}(L)+\left(2 \mu_{1}(L)\right)^{2}}\right\},
$$

and this result is best possible.

REMARK. We have $2 \mu_{1}>2 \tau \sqrt{\lambda_{1}^{2}+\left(2 \mu_{1}\right)^{2}}$ if and only if $2 \mu_{1}>\lambda_{1}\left(2 \tau / \sqrt{1-4 \tau^{2}}\right) \simeq$ $1.3711 \lambda_{1}$.

Theorem 2. If $K \in \mathcal{K}^{2}$ and $L \in \mathcal{L}^{2}$, with $G(K, L)=0$, then

$$
2 A(K)\left(\Delta(K)-2 \mu_{1}(L)\right) \leqslant \lambda_{1}(L) \Delta^{2}(K),
$$

and equality holds if and only if $K$ is a triangle with width $\Delta(K)$ and diameter $D(K)=$ $\left(\lambda_{1}(L) \Delta(K)\right) /\left(\Delta(K)-2 \mu_{1}(L)\right)$.

Corollary 1. Let $K \in \mathcal{K}^{2}$ and $L \in \mathcal{L}^{2}$ be given such that:

$$
\frac{A(K)}{D(K)}>k \max \left\{2 \mu_{1}, 2 \tau \sqrt{\lambda_{1}^{2}+\left(2 \mu_{1}\right)^{2}}\right\}, \quad k \in \mathbf{Z} .
$$

Then $G(K, L) \geqslant k^{2}$, that is, $\{K+\mathrm{g} \mid \mathrm{g} \in L\}$ is at least a $k^{2}$-fold covering of $E^{2}$.

Corollary 2. Let $K \in \mathcal{K}^{2}$ and $L \in \mathcal{L}^{2}$ be given such that:

$$
2 A(K)\left(\Delta(K)-2 \mu_{1}(L)\right)>\lambda_{1}(L) \Delta^{2}(K) .
$$

Then $K$ is a covering set.

\section{Proof of The Results}

Proof of TheOREM 1.

Theorem 1 will be proved by reducing the problem to rectangular lattices and symmetric convex bodies.

Let $\left\{\mathbf{b}_{1}, \mathbf{b}_{2}\right\}$ be a Minkowski reduced basis of $L$ (see $[\mathbf{2}, \mathbf{p . 8 4}]$ ), with $\left\|\mathbf{b}_{1}\right\|=\lambda_{1}(L)$ and let $\theta$ be the acute angle between $b_{1}$ and $b_{2}$ (so that $2 \mu_{1}(L)=\left\|b_{2}\right\| \sin \theta$ ).

Let $v_{1}=b_{1}$, and let $v_{2}$ be a vector of length $2 \mu_{1}$, perpendicular to $v_{1}$. Let $\Lambda$ denote the rectangular lattice determined by the basis vectors $v_{1}, v_{2}$. We shall prove the following:

Lemma 1. If $K$ is a convex body such that $G(K, L)=0$, then there exists another convex body $C$ containing no points of $\Lambda$, such that

(i) $A(C)=A(K), \quad D(C) \leqslant D(K)$,

(ii) $C$ is symmetric about the lines $x=1 / 2, y=1 / 2$, the coordinates $x$ and $y$ being relative to the basis $v_{1}, v_{2}$. 
Proof: Let $K^{\prime}$ be the convex body obtained from $K$ by symmetrisation with respect to the line $x=1 / 2$. It is well known that Steiner symmetrisation preserves convexity and areas, and does not increase diameters (see [1]). Therefore $K^{\prime}$ is convex, $A\left(K^{\prime}\right)=A(K)$, and $D\left(K^{\prime}\right) \leqslant D(K)$.

We shall show now that $G\left(K^{\prime}, \Lambda\right)=0$. If $K^{\prime}$ contained a lattice point of $\Lambda$, say the point $m v_{1}+n v_{2}$, then the line $y=n$, for the symmetry of $K^{\prime}$ with respect to $x=1 / 2$, intersects $K^{\prime}$ in a line segment of length greater than $\lambda_{1}$. The same line also intersects $K$ in a line segment of the same length and this implies that $G(K, L)>0$, contradicting the hypothesis. Therefore $G\left(K^{\prime}, \Lambda\right)=0$.

A similar argument shows that if we now symmetrise $K^{\prime}$ with respect to the line $y=1 / 2$, we obtain a convex body $C$ with the required properties.

In view of Lemma 1, to deduce the inequality of Theorem 1 it suffices to prove the following:

Lemma 2. Let $\Lambda$ be a rectangular lattice with basis $\left\{\lambda_{1} \mathbf{e}_{1}, \lambda_{2} \mathbf{e}_{2}\right\}$ (so that $\lambda_{i}(\Lambda)=\lambda_{i}$ where $\left.i=1,2\right)$. For any convex body $K$, symmetric with respect to the lines $x=\lambda_{1} / 2$ and $y=\lambda_{2} / 2$ with $G(K, \Lambda)=0$

$$
\frac{A(K)}{D(K)} \leqslant \max \left\{\lambda_{2}, 2 \tau \sqrt{\lambda_{1}^{2}+\lambda_{2}^{2}}\right\}
$$

and the inequality is sharp.

REMARK. For the original lattice $L$, Lemma 2 implies

$$
\frac{A(K)}{D(K)} \leqslant \max \left\{2 \mu_{1}(L), \lambda_{1}(L), 2 \tau \sqrt{\lambda_{1}^{2}(L)+\left(2 \mu_{1}(L)\right)^{2}}\right\}
$$

so that, by $2 \mu_{1}(L)>(\sqrt{3} / 2) \lambda_{1}(L)$, we obtain $2 \tau \sqrt{\lambda_{1}^{2}(L)+\left(2 \mu_{1}(L)\right)^{2}} \geqslant \tau \lambda_{1} \sqrt{7}>\lambda_{1}$.

Proof: To better utilise the symmetry of $K$, we translate the origin to the point $\left(\lambda_{1} / 2, \lambda_{2} / 2\right)$. Then the lattice $\Lambda$ is changed into the grid $\Gamma=\left\{\left(\lambda_{1}(m+1 / 2)\right.\right.$, $\left.\left.\lambda_{2}(n+1 / 2)\right) \mid m, n \in \mathbf{Z}\right\}$.

For the sake of brevity we write $D=D(K)$ and $A=A(K)$.

Since $K$ is centrally symmetric, it lies within the disc $x^{2}+y^{2} \leqslant D^{2} / 4$. If $D \leqslant$ $\sqrt{\lambda_{1}^{2}+\lambda_{2}^{2}}$, no point of $\Gamma$ is interior to this disc and then:

$$
A \leqslant \frac{\pi}{4} D^{2} \leqslant \frac{\pi}{4}\left(\sqrt{\lambda_{1}^{2}+\lambda_{2}^{2}}\right) D<2 \tau\left(\sqrt{\lambda_{1}^{2}+\lambda_{2}^{2}}\right) D .
$$

Therefore we may suppose $D>\sqrt{\lambda_{1}^{2}+\lambda_{2}^{2}}$.

Let $Q$ be the part of $K$ lying in the quadrant $x \geqslant 0, y \geqslant 0$. Because of the convexity of $K, Q$ lies below some line $l$ through the point $P=\left(\left(\lambda_{1} / 2\right),\left(\lambda_{2} / 2\right)\right)$ with 
non positive slope and with equation $y=\left(\lambda_{2} / 2\right)+m\left(x-\left(\lambda_{1} / 2\right)\right)$. Let us denote by $X$ and $Y$ respectively the intersection of the line $l$ with the coordinate axes and by $\mathcal{D}$ the disc $x^{2}+y^{2} \leqslant D^{2} / 4$. We distinguish two cases:

(a) $X, Y \notin \mathcal{D}$;

(b) exactly one of the points $X, Y$ is exterior to $\mathcal{D}$.

In case (a) the area of $Q$ is given by:

$$
A(Q)=\frac{\pi}{16} D^{2}-2 \int_{0}^{\sqrt{(D / 2)^{2}-q^{2}}}\left(\sqrt{(D / 2)^{2}-u^{2}}-q\right) d u
$$

where $q$ is the distance of the line $l$ from the origin.

We have thus:

$$
\frac{A}{D}=4 q \sqrt{1-\left(\frac{2 q}{D}\right)^{2}}+\frac{\pi}{4} D-2 D \int_{0}^{\sqrt{1-(2 q / D)^{2}}} \sqrt{1-t^{2}} d t
$$

and a short calculation shows that this function attains its maximum when $q=$ $(1 / 2) \sqrt{\lambda_{1}^{2}+\lambda_{2}^{2}}$, that is, when the line $l$ is normal to the segment $O P$, and the diameter $D$ satisfies the equation $\int_{0}^{\sqrt{1-(q / D)^{2}}} \sqrt{1-t^{2}} d t=\pi / 8$, that is, $D \simeq 1.09317 \sqrt{\lambda_{1}^{2}+\lambda_{2}^{2}}$, so that we obtain:

$$
\frac{A(K)}{D(K)}=2 \tau \sqrt{\lambda_{1}^{2}+\lambda_{2}^{2}}
$$

(Actually the function $A(K) / D(K)$ is an increasing function of $q$ and $q \leqslant$ $(1 / 2) \sqrt{\lambda_{1}^{2}+\lambda_{2}^{2}}$, moreover its derivative with respect to $D$ vanishes if and only if $D(K)$ satisfies the above equation.)

If $\lambda_{2} \leqslant 2 \tau \sqrt{\lambda_{1}^{2}+\lambda_{2}^{2}}$, the previous solutions are acceptable since for this value of $D(K)$ the points $X$ and $Y$ are exterior to the disk $\mathcal{D}$. Otherwise the maximum value of $A(K) / D(K)$ is taken when the line $l$ is normal to the segment $O P$ and passes through the point $Y$ : obviously in this case we have $A(K) / D(K)<2 \tau \sqrt{\lambda_{1}^{2}+\lambda_{2}^{2}}<\lambda_{2}$.

In case (b) let us suppose that $Y \in \mathcal{D}$, so $Q$ is a subset of the trapezium $T$ bounded by the coordinate axes, the line $l$ and the line $x=D / 2$. This trapezium has area $A(T)=(D / 8)\left[2 \lambda_{2}+m\left(D-2 \lambda_{1}\right)\right]$.

If $D \geqslant 2 \lambda_{1}$, this area is at most $\left(D(K) \lambda_{2}\right) / 4$ and thus

$$
\frac{A(K)}{D(K)}<\lambda_{2} \text {. }
$$

If $D<2 \lambda_{1}$, then $A(T)$ is an increasing function of $m$ so that it is easy to see that the maximum of the area of the region $Q$ is taken when the point $Y$ belongs to the line $l$.

In this case we have $A(K) / D(K) \leqslant 2 \tau \sqrt{\lambda_{1}^{2}+\lambda_{2}^{2}}$. 
REMARK. Inequality (7) could seem too wide, but the example of a rectangle with diagonal-length $D$ and an edge-length $\lambda_{2}$ shows that this bound is best possible when $D \rightarrow \infty$.

\section{PROOF OF THEOREM 2.}

For the sake of brevity we write $\Delta=\Delta(K), A=A(K), \lambda_{1}=\lambda_{1}(L)$ and $\mu_{1}=$ $\mu_{1}(L)$.

First we observe that the inequality in Theorem 2 can be written

$$
\frac{\lambda_{1}}{2 A}-\frac{\Delta-2 \mu_{1}}{\Delta^{2}} \geqslant 0
$$

Therefore we can take $K$ to be the set realising the minimum of the left-hand side of this inequality.

Because of $\Delta \leqslant 2 \mu_{1}+(\sqrt{3} / 2) \lambda_{1} \leqslant 4 \mu_{1}$ (see $\left.[10]\right),\left(\Delta-2 \mu_{1}\right) / \Delta^{2}$ is an increasing function of $\Delta$ and hence we choose $K$ with $A$ and $\Delta$ as large as possible.

It is clear that $K$ must be one of the following sets:

(a) a triangle with one (or two) of its sides on a lattice line;

(b) a triangle with one lattice point on each of its sides;

(c) a quadrilateral with one lattice point on each of its sides.

Moreover it is easy to see that in cases (a) and (c), $K$ circumscribes a parallelogram (which is a cell of $L$ ) with one side of length $\lambda_{1}$ and altitude $2 \mu_{1}$, and in case (b) $K$ circumscribes a triangle with one side of length $\lambda_{1}$ and relative altitude $2 \mu_{1}$.

Let $K$ be a triangle (cases (a) and (b)).

In this case we have

$$
A=\frac{1}{2} D \Delta \leqslant \frac{\lambda_{1} \Delta^{2}}{2\left(\Delta-2 \mu_{1}\right)}
$$

where the second inequality follows immediately from $\left(\Delta-2 \mu_{1}\right) D \leqslant \lambda_{1} \Delta$ proved in [11] and where equality holds if and only if $K$ is a triangle of width $\Delta(K)$ and diameter $D(K)=\lambda_{1}(L) \Delta(K) /\left(\Delta(K)-2 \mu_{1}(L)\right)$.

Thus it is sufficient to establish (2) in case (c).

Let $K$ be the quadrilateral $X Y Z T$ and let $O, B, C, E$ be lattice points such that $O \in[X, Y], B \in[Y, Z], C \in[Z, T], E \in[T, X], \overline{O B}=\overline{E C}=\lambda_{1}, \widehat{B O E}=\widehat{B C E}=$ $\varphi(\varphi \leqslant \pi / 2), \overline{O E} \sin \varphi=\overline{B C} \sin \varphi=2 \mu_{1}$. Let $m=\overline{T Y}$ and let $n$ be the length of the width of $K$ in the direction normal to $T Y$ and let us put $\overline{O E}=\nu$. Further let $\vartheta$ be the angle between the lines $E C$ and $X Z$.

By computing the area of $K$ and the areas of its component parts we obtain:

$$
2 A=m n= \begin{cases}\nu n \cos \vartheta+\lambda_{1} m \sin (\varphi-\vartheta) & \text { if } \vartheta \leqslant \pi / 2, \\ \nu n \cos \vartheta+\lambda_{1} m \sin (\varphi+\vartheta) & \text { if } \vartheta>\pi / 2\end{cases}
$$


As $\vartheta>\pi / 2$ implies $\varphi+\vartheta<\pi-\varphi$, we have $m n \leqslant \nu n+\lambda_{1} m \sin \varphi$ in either case, and equality holds if the line $X Z$ is parallel to the line $O B$. Thus we can suppose $2 A=m n=\nu n+\lambda_{1} m \sin \varphi$. Let $m \geqslant n$ so that $n \leqslant \lambda_{1} \sin \varphi+\nu$. Then

$$
2 A=m n=\frac{\nu n^{2}}{n-\lambda_{1} \sin \varphi} \leqslant \max \left\{\frac{\nu \Delta^{2}}{\Delta-\lambda_{1} \sin \varphi},\left(\lambda_{1} \sin \varphi+\nu\right)^{2}\right\} .
$$

Let $m<n$ so that $m \leqslant \lambda_{1} \sin \varphi+\nu$ and further let us suppose that the lines $X T$ and $Y Z$ are parallel or meet in the half-plane containing $Z$ and determined by $X Y$. (In the other cases the proof is similar.) Then $\Delta \leqslant m \sin (\widehat{X T Y}) \leqslant m \sin \varphi$. Since

$$
2 A=m n=\frac{m^{2} \lambda_{1} \sin \varphi}{m-\nu}
$$

is a decreasing function of $m$, then

$$
2 A \leqslant \max \left\{\frac{\nu \Delta^{2}}{\Delta-\lambda_{1} \sin \varphi},\left(\lambda_{1} \sin \varphi+\nu\right)^{2}, \frac{\lambda_{1} \Delta^{2}}{\Delta-\nu \sin \varphi}\right\} .
$$

Now it is a straightforward calculation to show that

$$
\max \left\{\frac{\nu \Delta^{2}}{\Delta-\lambda_{1} \sin \varphi},\left(\lambda_{1} \sin \varphi+\nu\right)^{2}, \frac{\lambda_{1} \Delta^{2}}{\Delta-\nu \sin \varphi}\right\}=\frac{\lambda_{1} \Delta^{2}}{\Delta-\nu \sin \varphi}
$$

so that Theorem 2 follows.

Proof of Corollaries.

As Corollary 2 is an obvious consequence of Theorem 2, we shall only prove Corollary 1 .

The idea of the proof follows an analogous argument given by Hammer in [3] (see also [11]) which we repeat here for completeness.

Let us suppose $k \geqslant 1$ (if $k=0$ the result is obvious) and consider the similarity transformation $K \rightarrow K^{\prime}=(1 / k) K$.

Obviously $A\left(K^{\prime}\right)=\left(1 / k^{2}\right) A(K)$ and $D\left(K^{\prime}\right)=(1 / k) D(K)$. Now let $\left\{\mathbf{b}_{1}, \mathbf{b}_{2}\right\}$ be a basis of $L$ with $\left|b_{i}\right|=\lambda_{i}$ and let $Q=q_{1} b_{1}+q_{2} b_{2}$ be a lattice point with $0 \leqslant q_{i} \leqslant$ $(k-1) \lambda_{i}(i=1,2)$. Then for the translate $K^{\prime \prime}$ of $K^{\prime}$ given by $K^{\prime \prime}=K^{\prime}-(1 / k) Q$ we have

$$
\frac{A\left(K^{\prime \prime}\right)}{D\left(K^{\prime \prime}\right)}=\frac{A\left(K^{\prime}\right)}{D\left(K^{\prime}\right)}=\frac{1}{k} \frac{A(K)}{D(K)}>\max \left\{2 \mu_{1}, 2 \tau \sqrt{\lambda_{1}^{2}+\left(2 \mu_{1}\right)^{2}}\right\}
$$

Thus, by Theorem $1, K^{\prime \prime}$ contains a lattice point $T$. Then $K^{\prime}$ contains the point $T+(1 / k) Q$, so that $K$ contains the point $U=k(T+(1 / k) Q)=k T+Q$. Since $Q$ can be chosen in $k^{2}$ different ways, by selecting each of $q_{1}, q_{2}$ in $k$ different ways we have $k^{2}$ distinct lattice points in $K$. 


\section{REFERENCES}

[1] H.G. Eggleston, Convexity (Cambridge University Press, New York, 1969).

[2] P. Erdös, P.M. Gruber and J. Hammer, Lattice points (Longman Scientific and Technical, - New York, 1989).

[3] J. Hammer, 'Lattice Points and Area-Diameter Relation', Math. Mag. 52 (1979), 25-26.

[4] R. Kannan and L. Lovàsz, 'Covering minima and lattice-points free convex bodies', Ann. of Math. 128 (1988), 577-602.

[5] J.C. Lagarias, H.W. Lenstra and C.P. Schnorr, 'Korkin-Zolotarev bases and successive minima of a lattice and its reciprocal lattice', Combinatorica 10 (1990), 333-348.

[6] U. Schnell and J.M. Wills, 'Two isoperimetric inequalities with lattice constraints', $M h$. Math. 112 (1991), 227-233.

[7] U. Schnell and J.M. Wills, 'On successive minima and intrinsic volumes', Mathematika 40 (1993).

[8] P.R. Scott, 'Area-diameter relations for two-dimensional lattices', Math. Mag. 47 (1974), . 218-221.

[9] P.R. Scott, 'Further Inequalities for convex sets with lattice point constraints in the plane', Bull. Austral. Math. Soc. 21 (1980), 7-12.

[10] S. Vassallo, 'A covering problem for plane lattices', Geom. Dedicata 43 (1992), 321-335.

[11] S. Vassallo and J.M. Wills, 'Covering sets for plane lattices', (preprint).

Università Cattolica del S. Cuore

Lárgo Gemelli, 1

I-20123 Milano

Italy 